Годишник Телекомуникации 2020, том 7, с. 29-39

Yearbook Telecommunications 2020, vol. 7, p. 29-39

eISSN 2534-854X

https://telecommunications.nbu.bg/bg/godishnik-telekomunikacii

DOI: https://doi.org/10.33919/YTelecomm.20.7.4

ВЪПРОСИ НА ОБЩАТА НАДЕЖДНОСТ НА СИСТЕМА SСАDА В КОРПОРАТИВНАТА ИНФРАСТРУКТУРА

Цветелина Симеонова

\title{
QUESTIONS OF THE OVERALL DEPENDABILITY OF THE SCADA SYSTEM IN THE CORPORATE INFRASTRUCTURE
}

\section{Tsvetelina Simeonova}

\begin{abstract}
Резюме: В работата се разглеждат особеностите на системата за управление на енергийните обекти на железопътната инфраструктура SCADA, както и въпроси на общата надеждност в корпоративната инфраструктура, което е цел на изследването. В изследването въз основа на приета структура и стойности на параметрите, се разглежда от какво зависи и как се променя коефициента на готовност на система SCADA при промяна на интензивността на възстановяване и при фиксирана интензивност на отказ. Резултатите показват, че при увеличаване на интензивността на възстановяване, коефициентите на готовност на така дефинираните телекомуникационна мрежа и система SCADA, нарастват, а същевременно намалява времето за престой.

За по-задълбочено изследване на общата надеждност на системата SCADA, е необходимо да се заложат конкретни нормативно приети надеждностни параметри на всички свързани елементи, както и реално зададена структура. Въз основа на това, че при осигурени ресурси за резервиране, може да се осигури дефинирана надеждност на системата при предварително зададени стойности на надеждностните параметри на нейните компоненти и структурата на резервирането; може да се реши също и обратната задача. Като принос на настоящата работа може да се отбележи предложения подход за изследване на надеждностното поведение на система SCADA в рамките на корпоративната инфраструктура и са направени изводи за практическото използване на резултатите.
\end{abstract}

Ключови думи: SCADA, надеждност, корпоративна инфраструктура, коефициент на готовност

\begin{abstract}
The paper considers the peculiarities of the SCADA (energy infrastructure management system in the railway infrastructure), as well as issues of the overall dependability in the corporate infrastructure, which is the aim of the study. The study, based on the accepted structure and values of the parameters, examines what depends on and how the readiness factor of a SCADA system changes when the recovery intensity changes and at a fixed failure rate. The results show that with increasing recovery intensity, the readiness factors of the telecommunications network and SCADA system thus defined increase, while reducing the downtime.

For a more in-depth study of the overall reliability of the SCADA system, it is necessary to set specific normatively accepted dependability parameters of all connected elements, as well as a real set structure. Based on the fact that with backup resources provided, a defined dependability of the system can be ensured at preset values of the reliability parameters of its components and the backup structure; the opposite problem can also be solved. The proposed approach for studying the reliable behavior of the SCADA system within the corporate infrastructure can be noted as a contribution of the present work and conclusions are made for the practical use of the results.
\end{abstract}

Keywords: SCADA, dependability, corporate infrastructure, readiness factor

\section{1. ВЪВЕДЕНИЕ}

Технологичното обновяване се налага периодично във всички отрасли на икономиката, а също и в жп инфраструктура, която по същество е сложна система, осигуряваща безопасното движение и експлоатация на жп транспорт. Комплексът от технологични средства на системите и подсистемите непрекъснато се обновява, поради изискванията за 


\section{ЦВЕТЕЛИНА СИМЕОНОВА}

по-висока ефективност при експлоатацията. Това се отнася както за преносната среда и съответните преносни системи, така и за оборудването осигуряващо събирането, обработката и разпределението на информацията, а също и за изпълнителните устройства. Приложението на информационните и комуникационни технологии са базира на обща платформа за мениджмънт, както и на системата за експлоатация и поддръжка на телекомуникационната мрежа O\&MS (Operational and Management System) и нейното управление от NMS (Network Management System). По подобен начин системата SCADA (Supervisory control and data acquisition) осигурява автоматизираното управление на енергийните обекти на жп инфраструктура. Особеностите на тяхната съвместна работа биха могли да бъдат основа за последващи процеси на интеграция [1].

Системата SCADA [2] като част от корпоративната жп инфраструктура служи за оперативно диспечерско управление на тяговите понижаващи подстанции, секционни разединители и основните секционни постове от Централен диспечерски центьр (ЦДЦ). За целта са предвидени съответни структура, функции и интерфейси на системата.

Общата принципна техническа структура на SCADA съдържа три йерархични нива:

- Обектно ниво - на което всички електросъоръжения в мрежата на SCADA ca обхванати от мрежа от програмируеми логически контролери PLC (programmable logic controller), които са конфигурирани по такъв начин, че да могат да обхващат всички информационни и управляеми точки. От операторски пулт на PLC на съответния обект може да се извършва неговото дистанционно управление [3].

- Комуникационно ниво - осъществява двупосочна комуникация между ЦДЦ и PLC на обекта по съответен протокол. Комуникацията между ЦДЦ и обектите се осъществява по обща за участька линия за връзка, която е резервирана.

- Диспечерско ниво - организирано е като високоскоростна Ethernet базирана локална мрежа.

Функциите на системата SCADA най-общо са обособени на три групи:

1. Процесни (външни) функции - за контрол и управление на електросъоръженията в подстанции, секционни постове и технологични съоръжения:

- събиране на динамична информация за текущото състояние на технологичните съоръжения и стойности на технологични параметри;

- подготовка и изпращане на информацията от сървърите към централния диспечерски центьр (ЦДЦ);

- онагледяване на получената в ЦДЦ информация;

- изпращане на управляващи команди от ЦДЦ към обектите и контрол на тяхното изпълнение.

2. Системни (вътрешни) функции - за контрол и управление на работата и състоянието на самата система

- поддържане на актуална база данни за всеки обект;

- поддържане на системно време и дата;

- вътрешен автоматичен контрол и диагностика на изправността на отделните устройства на системата;

- контрол за наличие на комуникация;

- локализиране на мястото и характера на повредата;

- контрол и диагностика на програмното осигуряване;

- формиране на алармени съобщения за появили се неизправности на обекта и в системата в процеса на работа.

3. Допълнителни функции

- генериране на протокол на събитията;

- генериране на алармени и неалармени съобщения; 
Годишник Телекомуникации 2020, том 7, с. 29-39

Yearbook Telecommunications 2020, vol. 7, p. 29-39

eISSN 2534-854X

https://telecommunications.nbu.bg/bg/godishnik-telekomunikacii

DOI: https://doi.org/10.33919/YTelecomm.20.7.4

- изработване на списьк на изпълнени и неизпьлнени команди;

- изработване на списък на квитирани и неквитирани алармени съобщения;

- осигуряване на възможност за преглед на данните в протоколите и списъците;

- съхраняване на данните от протоколите и списъците за време, което да може да се задава от потребителя на системата;

- извеждане на актуална за момента информация или в различен отрез от минало време при необходимост и по команда от оператора;

- разпечатване на различни протоколи и списьци по зададени от оператора критерии.

Системата SCADA също така е част от корпоративната телекомуникационна инфраструктура. Съвременните телекомуникационни мрежи трябва да изпълняват множество задачи извън рамките само на предаването на информация, а именно да поддържат и конвергенция на глас, видео и данни. Такива широколентови услуги могат да се предоставят на базата на съществуващите инфраструктури (SDH, ATM, Ethernet и др.), но постигането на ефективност при внедряването, администрирането и поддръжката изисква търсене на конкретни решения. Корпоративна мрежа с разпределени точки за достъп, с пропускателна способност до $10 \mathrm{Gbps}$, като цяло може да се реализира например чрез Ethernet и многопротоколна етикетна комутация MPLS (Multi-Protocol Label Switching). При такива условия, освен предаване на данни, може да се реализира предаване на глас по IP - VoIP (Voice over IP) с гарантиране на съответно качество на услугата QoS (Quality of Service), както и на видео по IP (за вътрешнокорпоративен обмен на информация и обучения или видеонаблюдение) [4].

\section{2. АСПЕКТИ НА ПОКАЗАТЕЛИТЕ ЗА НАДЕЖДНОСТ НА СИСТЕМА SCADА, КАТО ЧАСТ ОТ КОРПОРАТИВНАТА МРЕЖА [4]}

Най-важният аспект на телекомуникационната инфраструктура на корпоративната мрежа е изискването за много висока надеждност - с коефициент на готовност 0,99999. Постигането на тази норма изисква да се предвидят съществени допълнителни разходи при проектирането на мрежовите устройства и на мрежата като цяло. Такива разходи са свързани основно с различни варианти на резервиране/ дублиране и могат да бъдат например:

- Резервно разпределено токозахранване;

- Резервен таймер в реално време;

- Резервен модул за управление в „горещ” резерв;

- Резервен мрежов интерфейсен модул;

- Активни и пасивни карти за непрекъсваемо огледално приемане на данни;

- Автоматизирано предаване и приемане в случай на програмни или хардуерни грешки, а така също некоректни действия на потребителя;

- Активно обновяване на програмното осигуряване без прекъсване на работата;

- Мрежово управление както през външен канал, така и по мрежата.

Изискванията за отказоустойчивост (изразена чрез коефициент на готовност 0,99999) на хардуера и софтуера на мрежовите устройства (комутатор, маршрутизатор и др.) от своя страна налагат комплексно осигуряване на съвкупност от резервиране, управление, коефициент на грешки (с възможност за анализ на грешките), безопасност, готовност, околна среда, обслужване и поддръжка. Някои от техните особености ще се разгледат подолу. 


\section{ЦВЕТЕЛИНА СИМЕОНОВА}

Средно време на безотказна работа. Очакваното време за безотказна работа на компонентите се описва чрез параметъра средно време на безотказна работа MTBF (Mean Time Between Failures), чиято вероятностна стойност характеризира времето (в часове) до момента, в който даден компонент, блок или система се повредят. За коректно използване на MTBF е необходимо да се вземат предвид следните особености:

- MTBF се изчислява с отчитане на всички активни и пасивни конструктивни елементи на дадено устройство.

- Готовността се отчита на годишна база, което означава максимално време за престой под 5,2 мин. на годишна база.

Резервиране на източниците на електрозахранване. Резервирането на източниците на електрозахранване също е ключов показател, както при телекомуникационната мрежа, така и при системата SCADA. Телекомуникационните устройства обикновено работят със захранване 48 В постоянен ток, с преобразуване на 230 В променлив ток. Резервирането изисква осигуряване на два захранващи източника, резервиране на ниво блок или устройство (вкл. захранващата шина), използване на акумулаторни батерии, включване към управляващата система за подаване на аварийни сигнали и др.

Резервирани източници на времеви импулси. Друг ключов показател в цифровите системи е осигуряването на резервирани източници на времеви импулси за синхронизация при синхронните системи, т. нар. часовник в реално време RTC (Real-Time Clock). Основният системен източник на времеви импулси RTC се синхронизира независимо с всички синхронни устройства, както и с външните сигнали, с помощта на синхронизиращия мрежов протокол NTP (Network Time Protocol).

Примери и варианти за резервиране. Необходимата висока отказоустойчивост на системите и устройствата се осигурява чрез различни варианти на резервиране. Например комутаторите включват централен и резервен матричен контролер CFC (Central Fabric Controller), както и линийни интерфейсни карти LIC (Line Interface Card) - работещи в режим на „горещ” резерв. Контролерите се следят взаимно и в случай на открита грешка (несъвпадение на изходните команди), въз основа на автотест, се определя изправният контролер (без прекъсване на работата и падането на производителността), който продължава да функционира, а вторият се изолира до неговото възстановяване. Също така, пълно резервиране на свързаността на компонентите в комутатора се постига с независими вътрешни връзки между тях.

Резервиране на данните се осигурява с няколко механизма, синхронизирано между активния и резервния контролер:

- актуално съхраняване на конфигурационните данни във флаш-памет,

- статистиката, дългосрочните, както и динамичните изменения на данни (вследствие функционирането на системата OAM\&P (Operations, Administration, Maintenance \& Provisioning), огледално се предават на резервния контролер в реално време.

Превключването между основното и резервното оборудване е до $50 \mathrm{~ms}$.

Особености на надеждното функциониране на софтуера. Дублирането на данните намалява силно влиянието на хардуерните откази върху коефициента на готовност. При резервирането и предаването на данни в Ethernet ce използват различни стандартни механизми, вкл. и услуги от трети слой. Условията за надеждно функциониране на софтуера имат следните особености:

- Функционирането на отделните програмни модули се наблюдава от системния монитор, като в случай на открита неправилна работа той извършва превключване към резервното оборудване, с оповестяване посредством интерфейса за мениджмънт. 
Годишник Телекомуникации 2020, том 7, с. 29-39

Yearbook Telecommunications 2020, vol. 7, p. 29-39

eISSN 2534-854X

https://telecommunications.nbu.bg/bg/godishnik-telekomunikacii

DOI: https://doi.org/10.33919/YTelecomm.20.7.4

- Устройството поддържа съгласуваност в реално време на вграденото програмно осигуряване и всички динамични данни в контролерите.

- Обновяване на софтуера се извършва в основното устройство само когато изпълнението на неговите функции в конкретен времеви интервал може да поеме резервното устройство. Актуалната версия на софтуера се получава последно и от резервния контролер.

- Приемането на динамични данни (например актуализиране на маршрутните таблици чрез протокола OSPF (Open Shortest Path First)), както и динамичното „обновяване” на адресните таблици, се извършва без прекъсване на работата.

- Активирането на новото (или актуализираното) програмно осигуряване се извършва от системата за превключване между резервното и активното оборудване.

Мениджмънт на мрежата. Само превантивния и проактивен мениджмънт на мрежата позволява да се осигури необходимата отказоустойчивост на цялостната инфраструктура.

Ключов компонент за постигане на висока готовност при функционирането на мрежата е системата за мениджмънт на мрежата NMS (Network Management System), която осигурява разпознаването на всички активни мрежови устройства, техния мониторинг и мениджмънт, с използването на протокола за мениджмънт на мрежата SNMP (Simple Network Management Protocol). Например при мениджмънт на мрежата може да се използва пряко или непряко конфигуриране - назначаване на определена пропускателна способност на даден порт, предварително конфигуриране като стандартни профили на портовете, които физически още не съществуват и др.

Необходимо е също така да се отчитат и аспектите на сигурността, например автентификация на потребителите както на централната конзола, така и на активните компоненти; - при управление по външен канал или по мрежата да се прилага кодиране на данни и др.

Системата за експлоатация и поддръжка на мрежата включва следната функционалност:

- аларми от устройствата в реално време;

- автоматични тестове във фонов режим;

- текуща диагностика на хардуера и софтуера;

- текущо тестване на пътя на данните и на сигналите на управлението.

\section{3. ИЗСЛЕДВАНЕ НА ПОКАЗАТЕЛИ ЗА НАДЕЖДНОСТ НА СИСТЕМА SСАDА.}

На фиг. 1 е дадена примерна схема за изследване на надеждността на система SCADA (вкл. и NMS), а в табл. 1 е дадено описание на включеното оборудване от фиг. 1. На фиг. 2 е показано дърво на отказите, сьгласно схемата от фиг. 1.

Примерната схема показва структурата на системата за управление SCADA, на която комуникационното ниво е базирано на телекомуникационна мрежа, управлявана от NMS.

Интензивността на възстановяване на телекомуникационната мрежа (комуникационно ниво на система SCADA) е по-висока при наличие на NMS, тъй като функционалността на NMS поддържа готовността на мрежата. NMS е платформата върху която работи SCADA и зависи от нейната надеждност.

NMS няма критично влияние в случай на преконфигуриране на системата SCADA (при неизправност), като само „съобщава“ (подробно) на системния администратор за събитията в процеса на обслужване. 


\section{ВЬПРОСИ НА ОБЩАТА НАДЕЖДНОСТ НА СИСТЕМА SСАDА В КОРПОРАТИВНАТА ИНФРАСТРУКТУРА}

\section{ЦВЕТЕЛИНА СИМЕОНОВА}

При функционирането на система SCADA се разглеждат връзки от типа "от точка до точка"; това не променя общността на разглеждането, тъй като сървърите (на диспечерско ниво) осигуряват същия тип връзки за всеки активен процес, иницииран от програмируемите контролери PLC на обектно ниво.

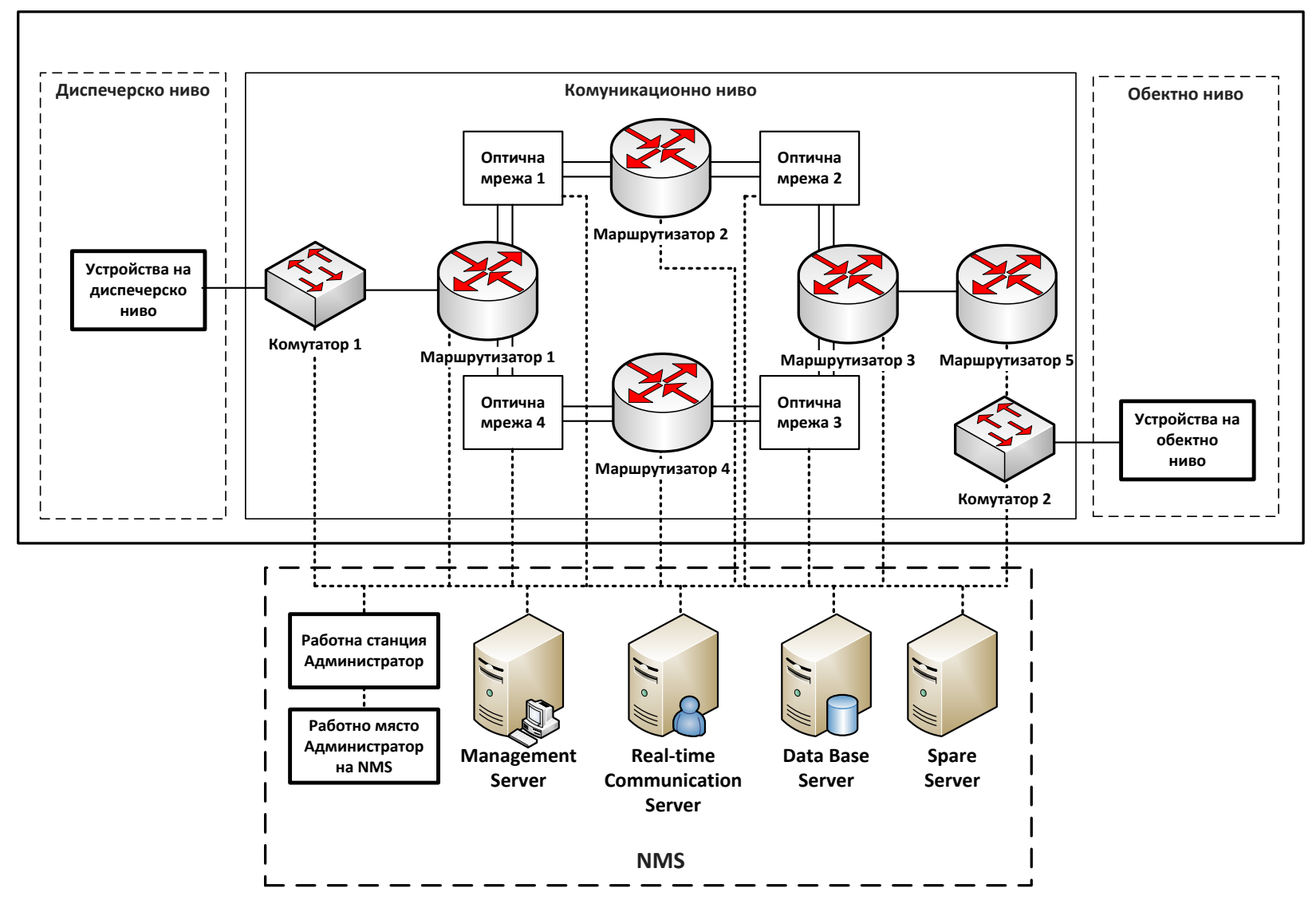

Фиг. 1. Примерна схема за изследване на надеждността на система SCADA (вкл. и NMS).

Таблица 1. Описание на включеното оборудване от фиг. 1.

\begin{tabular}{|c|c|}
\hline Ниво на SCADA & Описание на включеното оборудване \\
\hline 1. Диспечерско ниво & $\begin{array}{l}\text { сървър бази данни, комуникационен сървър, резервен сървьр, работна } \\
\text { станция диспечер 1, работна станция диспечер 2, работна станция } \\
\text { администратор на диспечерско ниво, работно място диспечер 1, работно } \\
\text { място диспечер 2, работно място администратор на диспечерско ниво } \\
\text { (сървъри). } \\
\text { Резервния сьрвър има съответния софтуер и може да резервира както сървъра } \\
\text { на бази данни, така и комуникационния сървър. Работна станция диспечер } 2 \text { и } \\
\text { работно място диспечер } 2 \text { резервира работна станция диспечер } 1 \text { и работно } \\
\text { място диспечер } 1 \text {. }\end{array}$ \\
\hline 2. Комуникационно ниво & $\begin{array}{l}\text { разклонителни и магистрални преносни линии, комутатори и } \\
\text { маршрутизатори. Магистралните оптични преносни линии работят във вид на } \\
\text { двоен ринг (резервиран). } \\
\text { Комутаторите и маршрутизаторите не се дублират, но са свързани към } \\
\text { двойния ринг така, че винаги да има поне два пътя на свързване между всеки } \\
\text { две точки. Основните компоненти на всеки от тях са резервирани. }\end{array}$ \\
\hline 3. Обектно ниво & $\begin{array}{l}\text { програмируеми контролери (PLC - Programmable Logical Controller), които не } \\
\text { са резервирани. Разглеждаме един контролер, но по принцип контролерите } \\
\text { обслужват всички обекти, контролирани от системата SCADA, като се } \\
\text { асоциират към всеки от тях. }\end{array}$ \\
\hline
\end{tabular}


Годишник Телекомуникации 2020, том 7, с. 29-39

Yearbook Telecommunications 2020, vol. 7, p. 29-39

eISSN 2534-854X

https://telecommunications.nbu.bg/bg/godishnik-telekomunikacii

DOI: https://doi.org/10.33919/YTelecomm.20.7.4

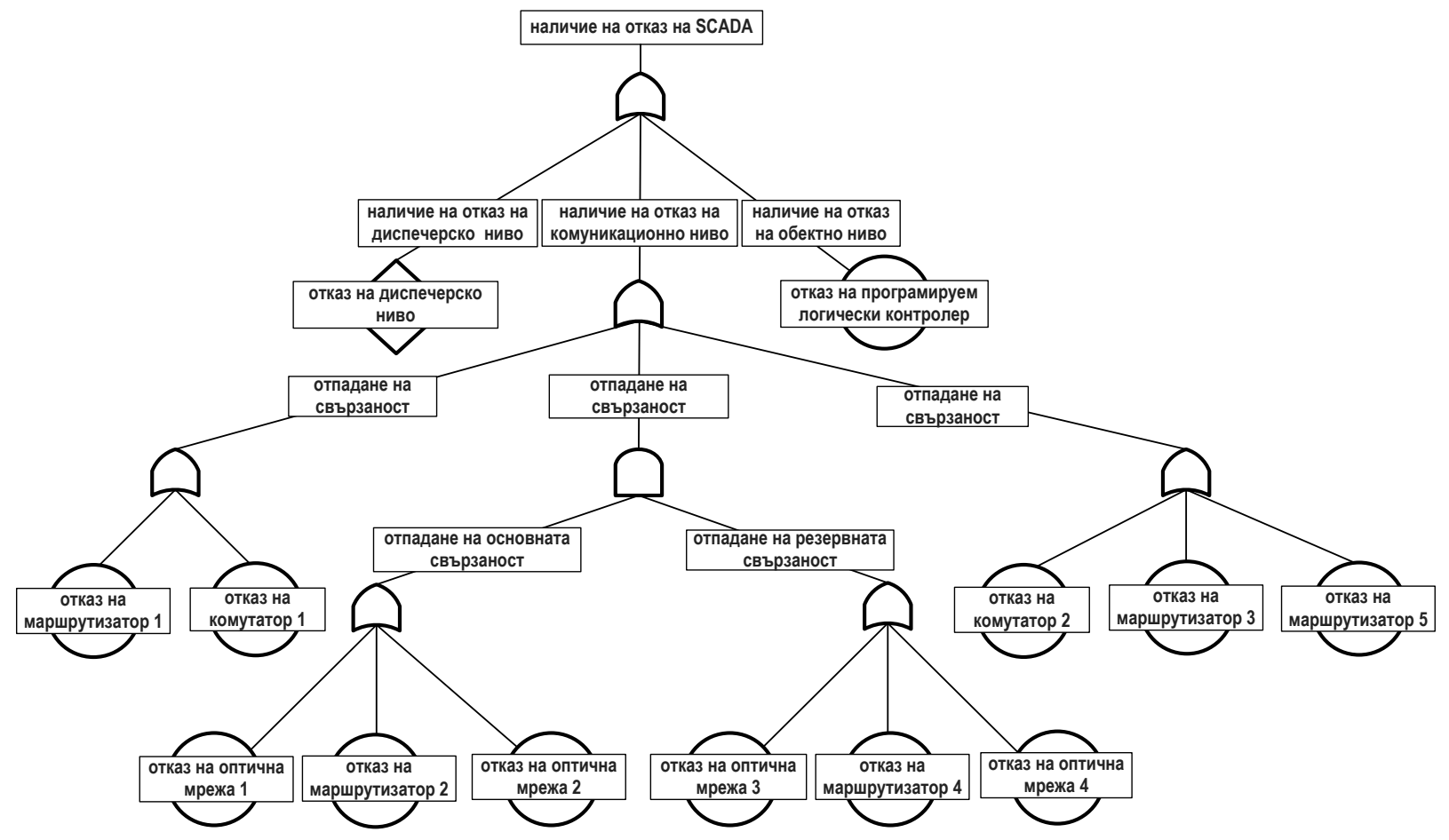

Фиг. 2. Дърво на отказите на примерна структура на системата SCADA.

Най-често постигането на висока степен на надеждност е чрез резервиране/дублиране на ниво компонент или устройство, което се отнася за съответния хардуер и софтуер, т.е. високата степен на надеждност се реализира чрез отказоустойчивост. Такъв подход с различни варианти на реализация се използва и при проектирането и изграждането на системата SCADA в жп инфраструктура. Благодарение на въведения по такъв начин излишък в структурата, въпреки възможни откази на компоненти, се постига отказоустойчивост, т.е. нарастване на надеждностните параметри вероятност за безотказна работа или коефициент на готовност.

Въз основа на показаната на фиг. 1 примерна схема, може да се изследва надеждността на системата, като, при заложени надеждностни параметри на елементите, се анализират приетите за възможни откази и тяхното влияние върху общата надеждност. Този подход е онагледен посредством дърво на отказите (фиг. 2), при отчитане на приетата структура от фиг. 1.

Методът дърво на отказите се основава на булевата алгебра и вероятностната логика и позволява качествен (логически) и количествен (вероятностен) анализ на надеждността и готовността на системата.

Използването на метода изисква задълбочено познаване на структурата и функционирането на техническата система, както и на надеждностните параметри на елементите. Отказите се моделират посредством символи, а логическите елементи отразяват отношението между тях, като за конкретния случай е отчетена структурата в надеждностно отношение. Съставеното дърво на отказите на фиг. 2 предполага определяне на коефициентите на престой (респективно средното време за престой).

Друг подход, с използване на надеждностна блокова диаграма, е също чрез метода на вероятностната логика, като означим надеждностно логическото състояние на елементите 


\section{ЦВЕТЕЛИНА СИМЕОНОВА}

на мрежата (работоспособно/ неработоспособно), понеже структурите са опростени, без риск да бъде пропуснат път за осъществяване на канал, могат да се запишат функциите на работоспособността. Разглежданата система е възстановима, и при преход към надеждностни функции, логическите променливи се заместват с коефициенти на готовност [5].

При анализа, и при двата подхода, може да се направят следните предположения [5]:

- Възможните повреди предполагат отчитане само отказ на оптична мрежа, като се приема, че отказите на другите линии (линиите за връзка основно на диспечерско и обектно ниво) се отнасят към общата надеждност на устройствата.

- Всички елементи на системата са възстановими (заменят се в процеса на функциониране). Характеристика на системата SCADA, представляваща интерес, е коефициентът на готовност в стационарен режим.

- Времевите параметри на системата с възстановяване след отказ са: интензивност на възстановяването и средно време между отказите MTBFs [5].

- Отказите и възстановяванията на отделните елементи са независими събития.

- Стационарно решение съществува, а дървото на отказите не съдържа повтарящи се откази.

- Параметри на всеки от елементите, показани на фиг. 1, са: K2 - коефициент на готовност; $\lambda$ - интензивност на отказ; $\mu$ - интензивност на възстановяване.

- Времето за безотказна работа и времето за възстановяване на всеки елемент са разпределени експоненциално с параметри $\lambda$ и $\mu$.

Въз основа на фиг. 1, коефициентът на готовност за система SCADA се дава от израза:

$\mathrm{K}_{\text {scada }}=\mathrm{K}_{\text {дн. }} \mathrm{K}_{\mathrm{M} 1} \cdot \mathrm{K}_{\mathrm{K} 1} \cdot\left[1-\left(1-\mathrm{K}_{\text {ом1 }} . \mathrm{K}_{\mathrm{M} 2} \cdot \mathrm{K}_{\text {ом2 } 2}\right) \cdot\left(1-\mathrm{K}_{\text {ом} 3} \cdot \mathrm{K}_{\mathrm{M} 4} \cdot \mathrm{K}_{\mathrm{oM} 4}\right)\right] \cdot \mathrm{K}_{\mathrm{K} 2} \cdot \mathrm{K}_{\mathrm{M} 3} \cdot \mathrm{K}_{\mathrm{M} 5} \cdot \mathrm{K}_{\text {плк }}$,

$\mathrm{K}_{\mathrm{net}}=\mathrm{K}_{\mathrm{M} 1} \cdot \mathrm{K}_{\mathrm{K} 1} \cdot\left[1-\left(1-\mathrm{K}_{\mathrm{oм} 1} \cdot \mathrm{K}_{\mathrm{M} 2} \cdot \mathrm{K}_{\mathrm{oM} 2}\right) \cdot\left(1-\mathrm{K}_{\mathrm{oм} 3} \cdot \mathrm{K}_{\mathrm{M} 4} \cdot \mathrm{K}_{\mathrm{oM} 4}\right)\right] \cdot \mathrm{K}_{\mathrm{K} 2} \cdot \mathrm{K}_{\mathrm{M} 3} \cdot \mathrm{K}_{\mathrm{M} 5}$,

$\mathrm{K}_{\text {scada }}=\mathrm{K}_{\text {дн }} \cdot \mathrm{K}_{\text {net }} \cdot \mathrm{K}_{\text {плк }}$,

където:

$\mathrm{K}_{\text {scada }}$ - коефициент на готовност за система SCADA;

$\mathrm{K}_{\text {net }}$ - коефициент на готовност на телекомуникационната мрежа;

$\mathrm{K}_{\text {дн }}$ - коефициент на готовност на диспечерско ниво;

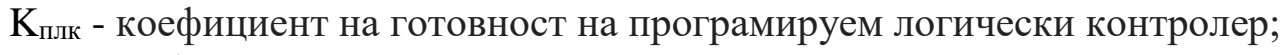

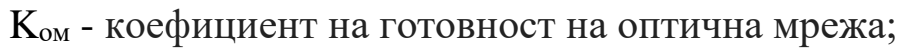

$\mathrm{K}_{\mathrm{M}}$ - коефициент на готовност на маршрутизатор;

$\mathrm{K}_{\mathrm{K}}$ - коефициент на готовност на комутатор.

При изследването се извършва определяне на коефициентите на готовност на телекомуникационната мрежа и на системата SCADA, а впоследствие и на средното време за престой - от съотношението (на годишна база): 8760 (1 - Кг мрежа); аналогично и за Кг на системата SCADA.

Приема се интензивността на отказ да е еднаква за мрежовите устройства, т.е. за всички маршрутизатори и комутатори, $\lambda=1 / 8760,1 /$ ч. (на годишна база).

За оптичната свързаност в опорната мрежа се приема Кг =0,99999 [6].

За диспечерско ниво (сървъри) и за неговата свързаност към мрежата се приема Кг = 0,99999, съгласно нормите за мрежи индустриален клас [6].

За обектно ниво (PLC) и за неговата свързаност към мрежата се приема Кг = 0,99995 [6]. Изследването разглежда от какво зависи и как се променя коефициента на готовност на система SCADA при промяна на интензивността на възстановяване и при фиксирана интензивност на отказ. 


\section{Годишник Телекомуникации 2020, том 7, с. 29-39}

\section{Yearbook Telecommunications 2020, vol. 7, p. 29-39}

По принцип, за целите на изследването, промяната на надеждността на система SCADA би могла да се оцени, като интензивностите на възстановяване на маршрутизаторите $\left(\mu_{\mathrm{r}}\right)$ и комутаторите $\left(\mu_{\mathrm{s}}\right)$ са равни или са различни.

В модела е прието, че устройствата са напълно резервирани (резервирани са техните блокове) и техните интензивности на възстановяване са равни. Приети са пет варианта на примерните експериментални данни, с оглед установяване на характера на изменение на общата надеждност на системата SCADA и получаване на графични зависимости. Направените пояснения са обобщени в табл. 2.

Таблица 2. Изходни данни и резултати за изследване на влиянието на надеждностните параметри на телекомуникационната мрежа $\left(\lambda, \mu_{\mathrm{r}}\right.$ и $\left.\mu_{\mathrm{s}}\right)$ върху надеждностните параметри (Кг, време за престой) на мрежата и на система SCADA.

\begin{tabular}{|c|c|c|c|c|c|}
\hline \multirow[b]{2}{*}{ Коефициент на готовност за: } & \multicolumn{5}{|c|}{$\lambda=0,00011415$} \\
\hline & $\begin{array}{l}\mu_{\mathrm{r}}=\mu_{\mathrm{s}}= \\
0,166667\end{array}$ & $\begin{array}{c}\mu_{\mathrm{r}}=\mu_{\mathrm{s}}= \\
0,2\end{array}$ & $\begin{array}{c}\mu_{\mathrm{r}}=\mu_{\mathrm{s}}= \\
0,25\end{array}$ & $\begin{array}{c}\mu_{\mathrm{r}}=\mu_{\mathrm{s}}= \\
0,3333\end{array}$ & $\begin{array}{c}\mu_{\mathrm{r}}=\mu_{\mathrm{s}}= \\
0,5\end{array}$ \\
\hline Маршрутизатори & 0,99931557 & 0,99942957 & 0,99954374 & 0,99965763 & 0,99977175 \\
\hline Комутатори & 0,99931557 & 0,99942957 & 0,99954374 & 0,99965763 & 0,99977175 \\
\hline Телекомуникационна мрежа & 0,99658203671 & 0,99715075443 & 0,99772055442 & 0,99828919066 & 0,99885920928 \\
\hline Време за престой, ч. & 29,94 & 24,96 & 19,96 & 14,98 & 9,99 \\
\hline Система SCADA & 0,99652224227 & 0,99709092588 & 0,99766069168 & 0,99822929379 & 0,99879927821 \\
\hline Време за престой, ч. & 30,46 & 25,48 & 20,49 & 15,51 & 10,51 \\
\hline
\end{tabular}

На фиг. 3 са показани зависимости на Кг от интензивността на възстановяване, а на фиг. 4 са показани зависимости на времето за престой от интензивността на възстановяване.

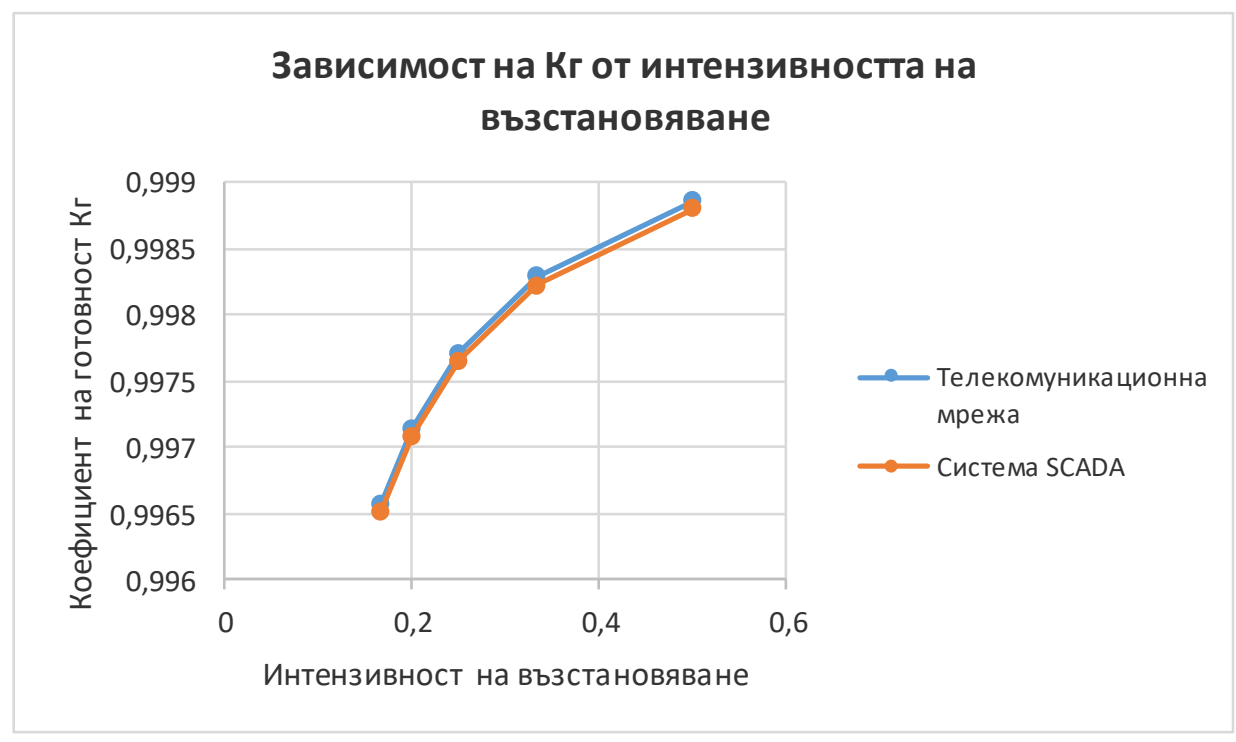

Фиг. 3. Зависимост на Кг от интензивността на възстановяване. 


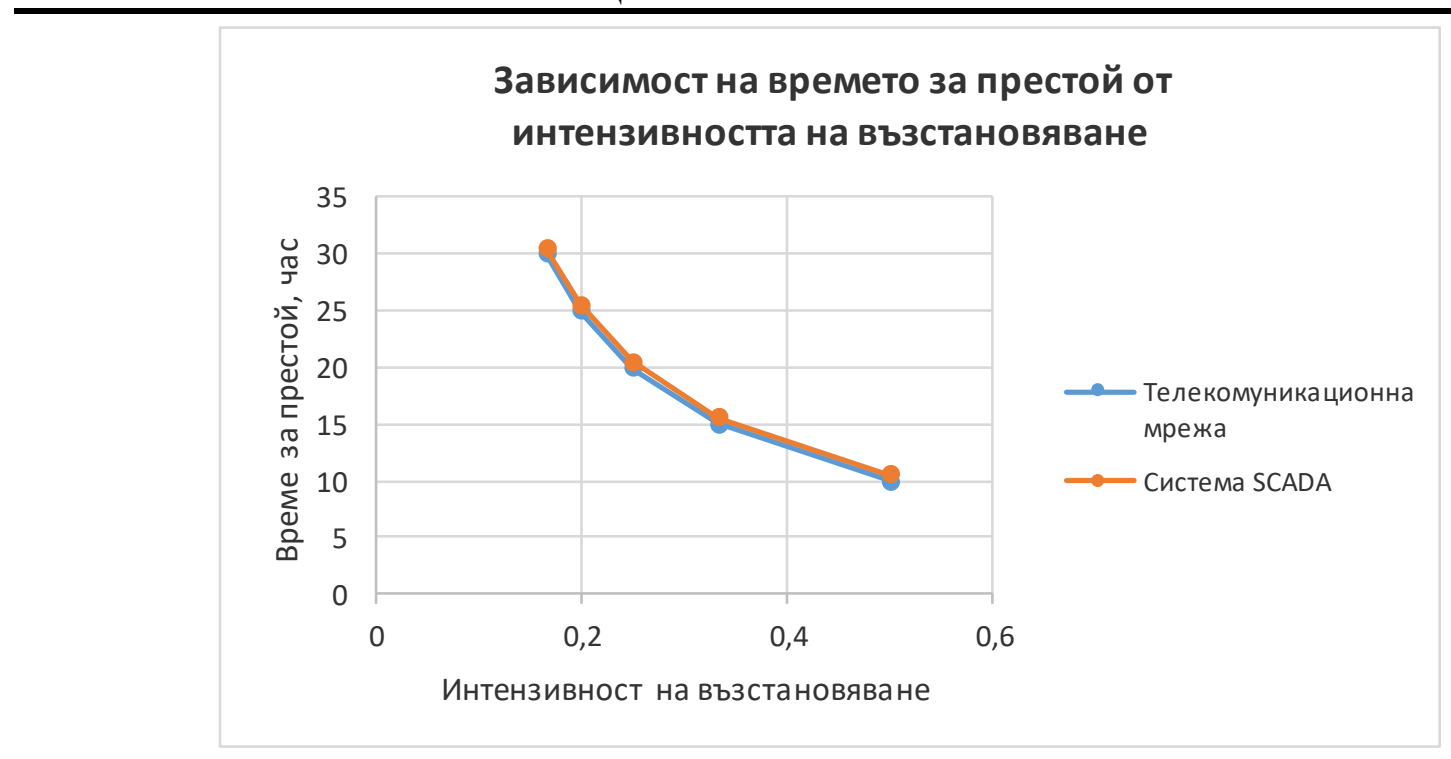

Фиг. 4. Зависимост на времето за престой от интензивността на възстановяване.

Характеристиките показват, че при увеличаване на интензивността на възстановяване, коефициентите на готовност на така дефинираните телекомуникационна мрежа и система SCADA, нарастват, което е очакван резултат. Същевременно намалява и времето за престой. Разликите в стойностите са минимални, като те се дължат на по-големия брой устройства на система SCADA.

\section{ЗАКЛЮЧЕНИЕ}

Може да се направят следните изводи:

1. При по-задълбочено изследване на общата надеждност на системата SCADA, e необходимо да се заложат нормативно приети надеждностни параметри на елементите, както и реално зададена структура.

2. Въз основа на това, че при осигурени ресурси за резервиране, може да се осигури дефинирана надеждност на системата при предварително зададени стойности на надеждностните параметри на нейните компоненти и структурата на резервирането; може да се реши също и обратната задача.

Като принос на настоящата работа може да се отбележи следното:

1. Предложен е подход за изследване на надеждностното поведение на система SCADA при съвместна работа с NMS.

2. Направени са изводи за практическото използване на резултатите.

\section{ЛИТЕРАТУРНИ ИЗТОЧНИЦИ (REFERENCES):}

[1] СИМЕОНОВА, Цветелина. Особености на съвместната работа на системите SCADA и NMS в жп инфраструктура. Механика, транспорт, комуникаџии. 2018, год. 16(3/2), с. 50-55. ISSN 1312-3823.;

Simeonova Tsvetelina. Specifics of simultaneous work of systems SCADA and NMS in the railway infrastructure.

“Mehanika, Transport, Komunikatsii”, ISSN 1312-3823, tom 16, broy 3/2, statia № 1739, 2018.

[2] Технически изисквания към елементите на железопьтната инфраструктура V02. ДП „Наџионална компания железопътна инфраструктура “ [онлайн]. ноември 2015 [прегледан 02 юни 2021]. Достьпен на: https://www.rail-infra.bg/; Tehnicheski iziskvania kam elementite na zhelezopatnata infrastruktura V02, DP "NKZhI", m. noemvri 2015.

[3] SIVANANDAM, S. N. Metro Traction Control System Using PLC And SCADA Monitoring. Gurukulam International Journal of Innovations in Science and Engineering. 2016, vol. 1(1), pp. 33-37. ISSN 2454-6631. [4] БУЮКЛИЕВ, Константин. Модернизаџия и оптимизиране на комуникаџиите чрез концентриране на глас и данни върху съществуваща IP мрежа [магистърска теза]. [онлайн]. София: СУ „Св. Климент Охридски“, 2007 [прегледан 02 юни 2021]. Достъпен на: https://research.uni-sofia.bg/handle/10506/206; 
Годишник Телекомуникации 2020, том 7, с. 29-39

Yearbook Telecommunications 2020, vol. 7, p. 29-39

eISSN 2534-854X

https://telecommunications.nbu.bg/bg/godishnik-telekomunikacii

DOI: https://doi.org/10.33919/YTelecomm.20.7.4

Buyukliev, K. Modernizatsia i optimizirane na komunikatsiite chrez kontsentrirane na glas i danni varhu sashtestvuvashta IP mrezha. 2007. [pregledan 22 fevruari 2019]. Dostapen na: https://research.unisofia.bg/handle/10506/206

[5] ХРИСТОВ, Христо и Венцислав ТРИФОНОВ. Надеждност и сигурност на комуникациите: Учебник за техническите университети. София: Нови знания, 2005. ISBN 954-931-543-6.; Hristov H., V. Trifonov, Nadezhdnost i sigurnost na komunikatsiite. Novi znania, Sofia, 2005.

[6] Проект за разширение на метрото в София, трета метролиния, първи етап - от км 4+950 до км 11+966,34. Доставка на метровлакове и изпълнение на системи за управление. Том 3. Технически спецификации и изисквания към системите, Част 3.2.: „Спецификация и технически параметри на транспортнокомуникационна система“. Метрополитен София [онлайн]. [прегледан 02 юни 2021]. Достъпен на: https://metropolitan.nit.bg/assets/resourcedocuments/1196/\%D0\%A2\%D0\%BE\%D0\%BC\%203\%20\%20\%D0\%A7\%D0\%B0\%D1\%81\%D1\%82\%203.2\%20\%20\%D1\%82\%D1\%80\%D0\%B0\%D0\%BD\%D1\%81\%D0 $\% \mathrm{BF} \% \mathrm{D} 0 \% \mathrm{BE} \% \mathrm{D} 1 \% 80 \% \mathrm{D} 1 \% 82 \% \mathrm{D} 0 \% \mathrm{BD} \% \mathrm{D} 0 \% \mathrm{BE}-$ \%D0\%BA\%D0\%BE\%D0\%BC\%D1\%83\%D0\%BD\%D0\%B8\%D0\%BA\%D0\%B0\%D1\%86\%D0\%B8\%D0\%BE\% D0\%BD\%D0\%BD\%D0\%B0\%20\%D1\%81\%D0\%B8\%D1\%81\%D1\%82\%D0\%B5\%D0\%BC\%D0\%B0.pdf; Proekt za razshirenie na metroto v Sofia, treta metrolinia, parvi etap - ot km 4+950 do km 11+966,34. Dostavka na metrovlakove i izpalnenie na sistemi za upravlenie. Tom 3 - Tehnicheski spetsifikatsii i iziskvania kam sistemite, Chast 3.2.: ,Spetsifikatsia i tehnicheski parametri na transportno-komunikatsionna sistema“. [pregledan 22 fevruari 2019]. Dostapen na:

https://metropolitan.nit.bg/assets/resourcedocuments/1196/\%D0\%A2\%D0\%BE\%D0\%BC\%203\%20$\% 20 \% \mathrm{D} 0 \%$ A7\%D0\%B0\%D1\%81\%D1\%82\%203.2\%20\%20\%D1\%82\%D1\%80\%D0\%B0\%D0\%BD\%D1\%81\%D0 $\% \mathrm{BF} \% \mathrm{D} 0 \% \mathrm{BE} \% \mathrm{D} 1 \% 80 \% \mathrm{D} 1 \% 82 \% \mathrm{D} 0 \% \mathrm{BD} \% \mathrm{D} 0 \% \mathrm{BE}-$

\%D0\%BA\%D0\%BE\%D0\%BC\%D1\%83\%D0\%BD\%D0\%B8\%D0\%BA\%D0\%B0\%D1\%86\%D0\%B8\%D0\%BE\% D0\%BD\%D0\%BD\%D0\%B0\%20\%D1\%81\%D0\%B8\%D1\%81\%D1\%82\%D0\%B5\%D0\%BC\%D0\%B0.pdf

\section{Информация за автора:}

ac. д-р инж. Цветелина Симеонова, Катедра "СОТС” при ВТУ "Т. Каблешков", ул. Гео Милев № 158, 1406, Тел.: 0885858022, e-mail: ts.b.simeonova@abv.bg

\section{Contacts:}

Assist. Prof. Tsvetelina Simeonova, PhD, 1406, Department Communication and security equipment and systems, University of Transport "T. Kableshkov", 158 Geo Milev St., Sofia, Tel.: (359) 885858022, e-mail: ts.b.simeonova@abv.bg

Дата на постьпване на ръкописа (Date of receipt of the manuscript): 13.07.2020. Дата на приемане за публикуване (Date of adoption for publication): 27.09.2020. 